Article

\title{
Characteristics of $\mathrm{CO}_{2}$ Hydrate Formation and Dissociation in Glass Beads and Silica Gel
}

\section{Mingjun Yang ${ }^{1}$, Yongchen Song ${ }^{1, *}$, Xuke Ruan ${ }^{1}$, Yu Liu ${ }^{1}$, Jiafei Zhao ${ }^{1}$ and Qingping Li ${ }^{2}$}

1 Key Laboratory of Ocean Energy Utilization and Energy Conservation of Ministry of Education, Dalian University of Technology, Dalian 116024, China; E-Mails: yangmj@dlut.edu.cn (M.Y.); ruancoco@126.com (X.R.); liuyu@dlut.edu.cn (Y.L.); jfzhao@dlut.edu.cn (J.Z.)

2 China National Offshore Oil Corporation Research Center, Beijing 100027, China; E-Mail: liqp@cnooc.com.cn

* Author to whom correspondence should be addressed; E-Mail: songyc@dlut.edu.cn; Tel.: +86-411-84706722; Fax: +86-411-84708015.

Received: 9 February 2012; in revised form: 21 March 2012 / Accepted: 6 April 2012 / Published: 16 April 2012

\begin{abstract}
CO}_{2}$ hydrate formation and dissociation is crucial for hydrate-based $\mathrm{CO}_{2}$ capture and storage. Experimental and calculated phase equilibrium conditions of carbon dioxide $\left(\mathrm{CO}_{2}\right)$ hydrate in porous medium were investigated in this study. Glass beads were used to form the porous medium. The experimental data were generated using a graphical method. The results indicated the decrease of pore size resulted in the increase of the equilibrium pressure of $\mathrm{CO}_{2}$ hydrate. Magnetic resonance imaging (MRI) was used to investigate the priority formation site of $\mathrm{CO}_{2}$ hydrate in different porous media, and the results showed that the hydrate form firstly in BZ-02 glass beads under the same pressure and temperature. An improved model was used to predict $\mathrm{CO}_{2}$ hydrate equilibrium conditions, and the predictions showed good agreement with experimental measurements.
\end{abstract}

Keywords: $\mathrm{CO}_{2}$ hydrate; porous medium; equilibrium condition

\section{Introduction}

The greenhouse effect is leading to a significant climate warming and weather changes [1]. $\mathrm{CO}_{2}$ is considered to be one of the most important greenhouse gases, and the disposition of $\mathrm{CO}_{2}$ has become an issue of worldwide concern [2]. International Energy Agency (IEA) proposed that if the target of 
climate change control was obtained without $\mathrm{CO}_{2}$ capture and storage (CCS), the total cost will increase $70 \%$ more than with CCS by 2050 [3].

Gas hydrate technology is a new subject based on ice-like crystalline compounds where gas molecules are held within cavities formed by water molecules [4], and it is developed and used in some industrial field recently, including refrigeration, gas storage and transportation, gas separation. Hydrate-based $\mathrm{CO}_{2}$ separation as a promising option for fossil fuel power plant $\mathrm{CO}_{2}$ capture (the first step of CCS) is attracting people's attention, which is a novel concept that aims to use $\mathrm{CO}_{2}$ hydrate to trap $\mathrm{CO}_{2}$ molecules in a lattice of water molecules [5]. The other proposed scheme is to sequester $\mathrm{CO}_{2}$ in form of gas hydrates in ocean and marine sediment (the last step of CCS) [6]. To make $\mathrm{CO}_{2}$ hydrate formation quick and economical during the capture process, or stabilized in marine sediments, it is necessary to understand the thermodynamic characters for $\mathrm{CO}_{2}$ hydrate formation and dissociation, especially the phase equilibrium conditions.

$\mathrm{CO}_{2}$ hydrate equilibrium conditions have been widely investigated. Wendland et al. [7], Yang et al. [8], Englezos et al. [9], Breland et al. [10], Dholabhai et al. [11,12], Kang et al. [13], Mohammadi et al. [14] investigated the $\mathrm{CO}_{2}$ hydrate phase equilibrium in water with different additives. The studies concerning $\mathrm{CO}_{2}$ hydrate equilibrium in porous medium are also familiar. Handa and Stupin [15] observed the shift that hydrates capillary equilibria from that in the bulk water. Uchida, et al. [16] likewise observed an equilibria shift in silica glass with pores of radii $100 \AA, 300 \AA$, and $500 \AA$, and they estimated that the apparent interfacial free energy between methane hydrates and water in the confined condition was approximately $3.9 \times 10^{-2} \mathrm{~J} \cdot \mathrm{m}^{-2}$. At the same time, Clennell et al. [17] and Clarke et al. [18] also concluded that observed hydrate equilibrium shifts in the ocean floor were caused by capillary effects in small sediment pores. Zatsepina et al. [19] measured $\mathrm{CO}_{2}$ hydrate stability in porous media. They concluded that when the vapor phase of $\mathrm{CO}_{2}$ was absent, the volume of hydrate was limited by the transport of $\mathrm{CO}_{2}$ from solution. Smith et al. [20] measured equilibrium pressures for $\mathrm{CO}_{2}$ hydrate in silica gel pores with nominal radii of 7.5, 5.0, and $3.0 \mathrm{~nm}$, and observed they were higher than those for $\mathrm{CO}_{2}$ hydrate in bulk water. Anderson et al. [21] presented experimental methane, carbon dioxide, and methane-carbon dioxide hydrate equilibrium and ice-melting data for meso-pores silica glass, and determined similar values of interfacial tensions for ice-water, methane clathrate-water, and carbon dioxide clathrate-water. Following their studies, Kumar [22] collected experimental equilibrium conditions data for $\mathrm{CO}_{2}$ hydrate in porous medium and measured the permeability of the porous medium in the presence of hydrate by flowing through the system. Turner et al. [23] reported that any shift in pores larger than $600 \AA$ in radius cannot be distinguished from errors of the thermocouples in their equilibrium apparatus (with thermocouple error of $\pm 0.5 \mathrm{~K}$ ). Aladko et al. [24] investigation hydrates equilibrium of ethane, propane, and carbon dioxide dispersed in silica gel meso-pores at pressures up to $1 \mathrm{GPa}$. The result showed that the experimental dependence of hydrate decomposition temperature on the size of pores can be described on the basis of the Gibbs-Thomson equation only if one takes into account changes in the shape coefficient that is present in the equation.

In this work, considering the limited data available in macro porous medium, we carried out experiments in glass beads which enable the study of the impact of porosity-related properties like capillary effects on the equilibrium conditions. The characteristics of $\mathrm{CO}_{2}$ hydrate formation and dissociation in porous medium were investigated by experimental observations and numerical 
modeling. MRI was also used in this study to determine the priority formation position of $\mathrm{CO}_{2}$ hydrate in different pore sizes.

\section{Experimental}

\subsection{Experimental Apparatus and Materials}

The experimental apparatus used in this study is shown in Figure 1 and for further details of the experimental apparatus the reader can be referred to the previous publications of our research team [25,26]. A high-pressure resistant vessel made of 316-stainless steel with a volume of $476 \mathrm{~mL}$ is used as the reactor. Thermocouples (produced by Yamari Industries, Osaka, Japan) and two pressure transducers (produced by Nagano Keiki, Tokyo, Japan) are connected to the vessel. The estimated errors of temperature and pressure measurements are $\pm 0.1 \mathrm{~K}$ and $\pm 0.1 \mathrm{MPa}$, respectively. Glass beads (produced by As-One Co., Ltd., Japan) and silica gel (produced by Anhui Liangchen Silicon Material Co. Ltd., Huoshan, Anhui, China) were used to form porous medium. $\mathrm{CO}_{2}$ (mass fraction 0.999) was provided by Dalian Guangming Special Gas Co. Ltd., China. Table 1 summarizes the specifications of all the components. All the chemicals were unpurified and the de-ionized water was used in all the experiments.

Figure 1. Scheme of the gas hydrate experimental apparatus.

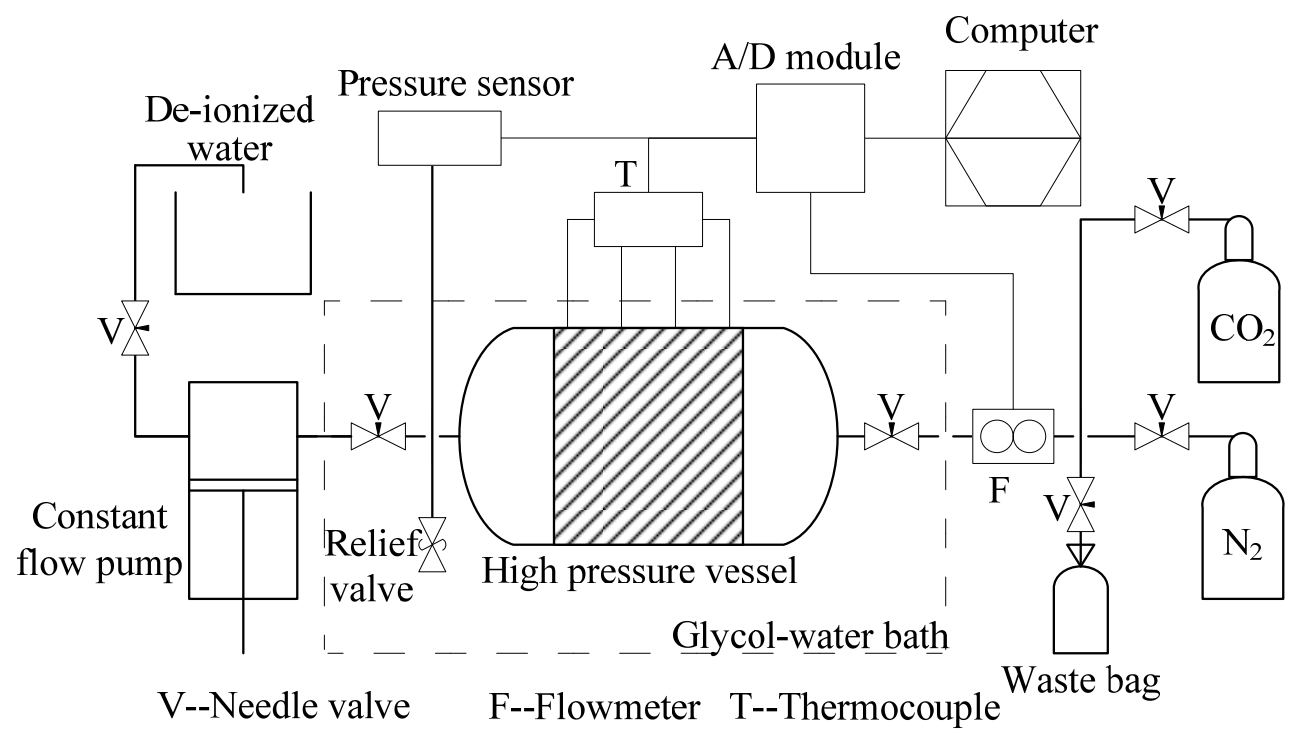

Table 1. Property and supplier of materials.

\begin{tabular}{lllc}
\hline Material & Purity/Composition & Particle size & Supplier \\
\hline $\mathrm{CO}_{2}$ & $99.9 \%$ & - & Dalian Guangming special gas Co., Ltd., China \\
$\mathrm{BZ}-01$ & Soda glass & $0.105-0.125 \mathrm{~mm}$ & As-One Co., Ltd., Japan \\
$\mathrm{BZ}-02$ & Soda glass & $0.177-0.250 \mathrm{~mm}$ & As-One Co., Ltd., Japan \\
$\mathrm{BZ}-04$ & Soda glass & $0.350-0.500 \mathrm{~mm}$ & As-One Co., Ltd., Japan \\
Mix glass beads & Soda glass and clay & $0.125-1.0 \mathrm{~mm}$ & Self-made \\
Silica gel & Silica gel & $0.42-0.84 \mathrm{~mm}$ & Anhui Liangchen Silicon Material Co., Ltd., \\
& & & China (mean particle pore size 8.0-10 nm) \\
\hline
\end{tabular}




\subsection{Experimental Procedures}

The graphical method was used to measure hydrate phase equilibrium conditions by keeping one of the three parameters of pressure $(p)$, volume $(V)$ and temperature $(T)$ constant and changing one of the remaining parameters to form or decompose the hydrate. In this study, experiments were carried out using the graphical method by keeping volume constant. $\mathrm{CO}_{2}$ hydrate was formed and decomposed by cooling and heating the closed system (volume constant). Neither gas nor water was added to the system during each experimental cycle.

Dry glass beads were tightly packed into the vessel with de-ionized water to simulate the porous medium. Then the vessel was reconnected to the system, and $\mathrm{CO}_{2}$ was injected to discharge the water partly from the vessel to obtain residual water saturation. After the outlet valve was closed, $\mathrm{CO}_{2}$ was slowly injected continuously into the vessel to a designated pressure and the pressure was kept constant. The amounts of residual water and injected $\mathrm{CO}_{2}$ were all recorded. When the temperature was steady and there were no leaks, the temperature was decreased. Once a temperature increase appeared, we confirmed that $\mathrm{CO}_{2}$ hydrates were formed in the vessel due to the exothermic (hydrate formation) reaction. The formation process finished when there was no pressure change. Then the bath was warmed slowly to dissociate the $\mathrm{CO}_{2}$ hydrate. The pressure and temperature $(p-T)$ conditions at the end of the hydrate decomposition was considered to be $\mathrm{CO}_{2}$ hydrate phase equilibrium conditions.

\section{Results and Discussion}

\section{1. $\mathrm{CO}_{2}$ Hydrate Formation and Dissociation Process}

The $p$ - $T$ curve during $\mathrm{CO}_{2}$ hydrate formation and dissociation was dependent on the kinds of porous medium. Two cases of $\mathrm{CO}_{2}$ hydrate formation process were examined experimentally (only the vapor $\mathrm{CO}_{2}$, initial state, was discussed in this study). The $p-T$ curve when hydrate formed in the BZ-01, BZ-02, BZ-04 is shown in Figure 2.

Figure 2. Typical $p$ - $T$ curves for $\mathrm{CO}_{2}$ hydrate formation in BZ-01, BZ-02 and BZ-04 glass beads with uniform pore size distribution.

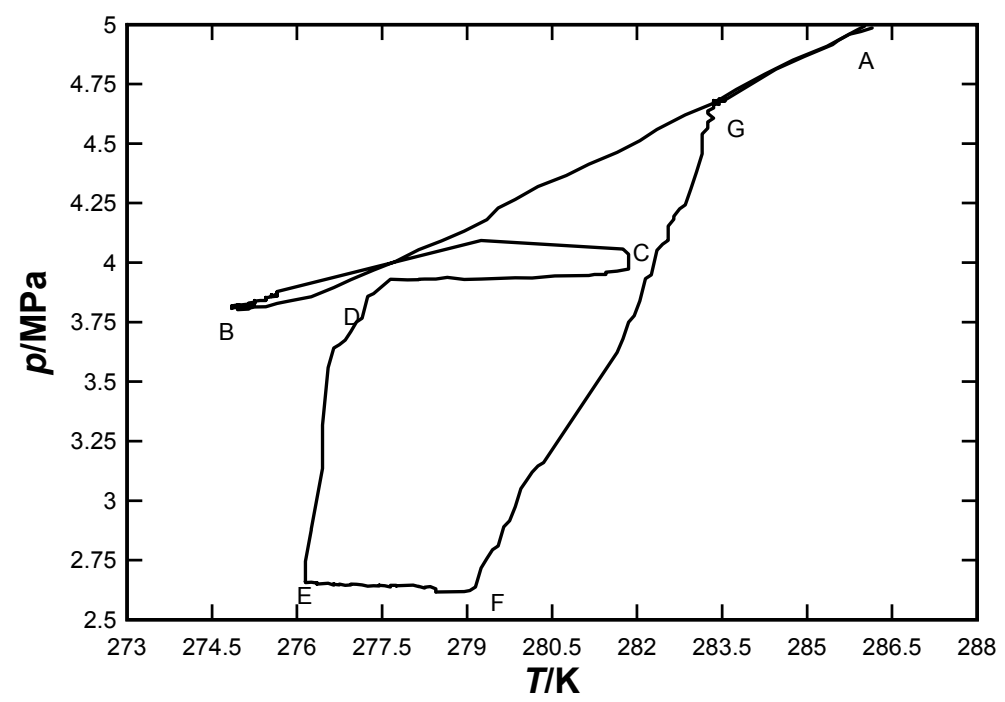


In this case, the pore size of porous medium was approximately uniform, and there is only one hydrate formation stage, point B-E. After the bath temperature was decreased to the target value, which was usually more than $4 \mathrm{~K}$ below the estimated equilibrium temperature (A-B), the sample temperature rose suddenly later $(\mathrm{B}-\mathrm{C})$, which was caused by $\mathrm{CO}_{2}$ hydrate formation, and the pressure soon decreased (due to gas consumption as the gas was encaged in the hydrate lattice). Since the $\mathrm{CO}_{2}$ hydrate formation rate was low and there was a high temperature difference between the porous medium and bath, the temperature decreased slowly (C-D). When the $\mathrm{CO}_{2}$ hydrate formation process finished, the temperature decreased down to the initial setting value (D-E). Then the vessel was warmed gradually, when the $p-T$ condition reached to $\mathrm{F}$, the hydrate began to decompose, which caused a significant pressure increase (F-G). Point $G$ was considered as the end of hydrate decomposition, which implied the equilibrium condition for this case. After intersecting with A-B, the $p$ - $T$ curve was back to point A along the temperature reduction process.

When $\mathrm{CO}_{2}$ hydrate formed in mixed glass beads and silica gel, the hydrate formation process can be divided into two phases, as shown in Figures 3 and 4. This was caused by the different kinds of pore sizes present in these pores medium. Figure 3 showed the experiment carried out in mixed glass beads. The first formation stage (A-E) was the same as that discussed for Figure 2. In this stage, $\mathrm{CO}_{2}$ hydrate may form in bigger or smaller size pores, the conclusion could not be educed. A significant pressure drop and temperature rise caused by the formation of a big amount of hydrates were observed from $B$ to $\mathrm{C}$. Since the thermostat temperature was still low, the temperature will decrease to the set value due to the heat transfer (C-D). After that, the second formation stage occurred at E-F, where temperature also showed a dramatic increase. In this stage, hydrate formed in the other sized pores. In order to determine the first $\mathrm{CO}_{2}$ hydrate formation site, MRI was used in this work. The experimental procedure and results are discussed in the following paragraph.

Figure 3. Typical $p-T$ curves for $\mathrm{CO}_{2}$ hydrate formation in mixed glass beads with non-uniform pore size distribution.

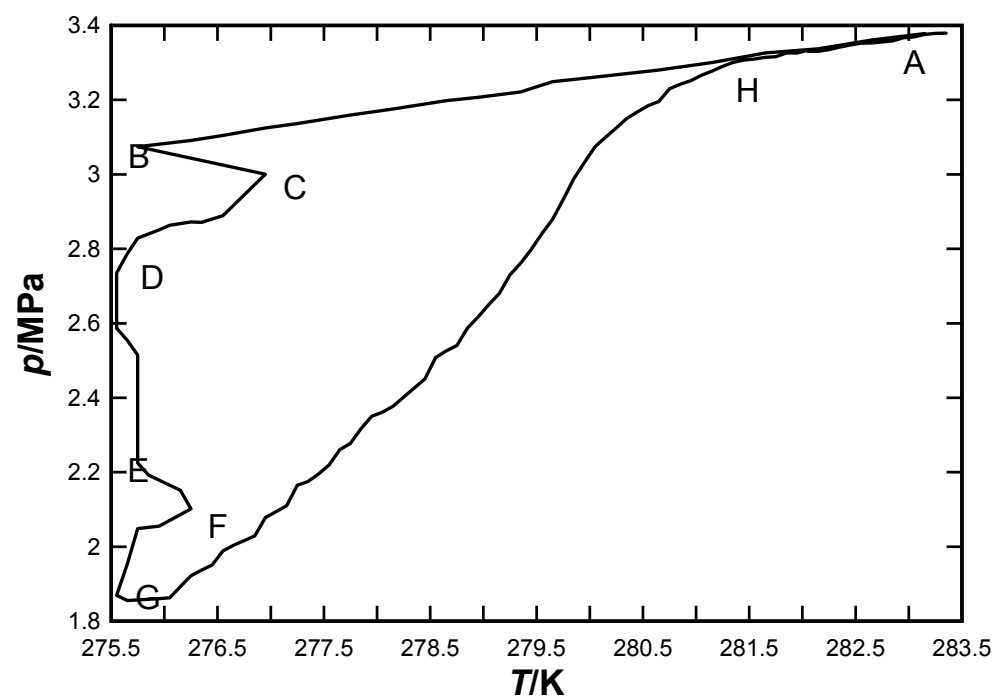

When silica gel was used in the experiments, there are two size pores in the sediment, the larger pore is inter-particle porosity and the smaller one is the inherent pore size of silica gel. The hydrate formation process was shown in Figures 4 and 5, and the notes (A-G) in them correspond with each 
other. Once the pressure had dramatically decreased (B-C-D, in Figure 4), the hydrate began to nucleate and grow (the first formation stage), and the temperature showed a small increase during this time. Then the pressure remained constant and the temperature decreased further (D-E). As the temperature reached $273.6 \mathrm{~K}$, the pressure decreased dramatically again $(\mathrm{E}-\mathrm{F})$, which meant the second formation stage of $\mathrm{CO}_{2}$ hydrate. The hydrate dissociation process was the same as that in Figures 2 and 3. In this case, the first hydrate formation site was also uncertain. Usually, we proposed that the $\mathrm{CO}_{2}$ hydrate formed firstly in bigger pores, because the two formation stage occurred at $1.95 \mathrm{MPa}$ and $275 \mathrm{~K}$, and $1.75 \mathrm{MPa}$ and $273 \mathrm{~K}$, respectively, during the formation process. The sub-cool temperature of second stage was high than the first one, which was the same as the equilibrium condition of hydrate in different size pores. The smaller the pore size corresponds to the lower hydrate equilibrium temperature at the same pressure.

Figure 4. Typical $p$ - $T$ curves for $\mathrm{CO}_{2}$ hydrate formation in silica gel with non-uniform pore size distribution.

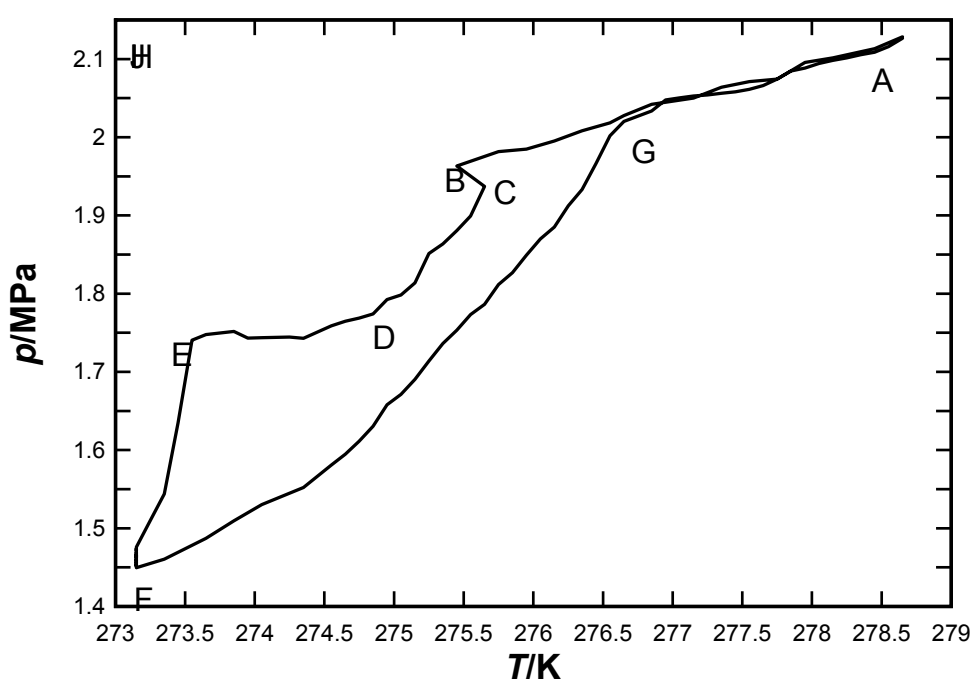

Figure 5. Typical curves of pressure and temperature changes with time for $\mathrm{CO}_{2}$ hydrate in the mixed glass beads and silica gel: the broken line represents the value of pressure and the solid line indicates the temperature in the vessel.

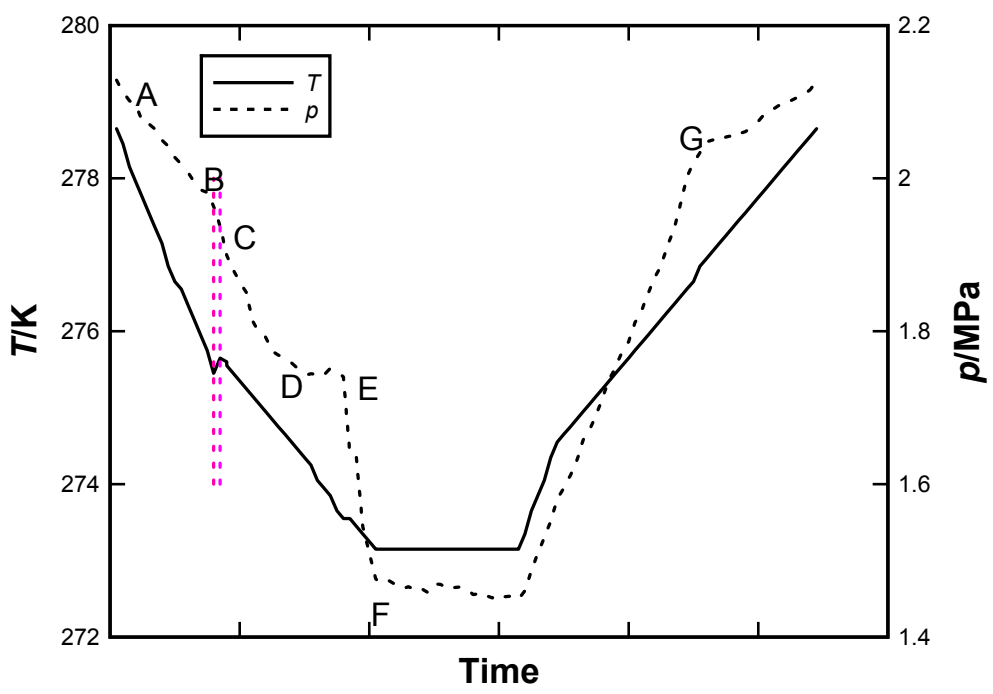




\subsection{Determination of $\mathrm{CO}_{2}$ Hydrate Priority Formation Site Using MRI}

MRI was used to test the hypothesis that $\mathrm{CO}_{2}$ hydrate formed firstly in the bigger pores. In order to get close to the study conditions, the different pore sizes were built up with BZ-01 and BZ-02 glass beads. During the experiments, BZ-01 glass beads were packed into the vessel tightly with de-ionized water firstly. The height of BZ-01 was about half of the vessel. Then a plastic slice was put into the vessel, which was used to divide the two glass kinds of beads. Then BZ-02 was packed into the vessel fully. The other procedure was similar with that discussed before, the main difference being that the vessel was put into the MRI to obtain images during the formation process. The schematic diagram of the MRI apparatus and the distribution of glass beads in the high pressure vessel were shown in Figure 6. The experimental apparatus consisted of a high-pressure vessel, MRI system, data acquisition system, high-pressure pumps, and a low-temperature cooling system. The vessel is made of polyimide which is a non-magnetic material, and its design pressure is $12 \mathrm{MPa}$. The effective size for packed glass beads is $\Phi 15 \times 200 \mathrm{~mm}$. The MRI (Varian) was operated at a resonance of $400 \mathrm{MHz}, 9.4 \mathrm{Tesla}$, to measure hydrogen. The high-precision thermostat bath (F25-ME, produced by Julabo Labortechnik $\mathrm{GmbH}$, Germany) filled with fluorocarbon (FC-40, supplied by 3M Company, USA) was used to control temperature precisely. ${ }^{1} \mathrm{H}-\mathrm{MRI}$ produces images of hydrogen contained in liquids, but does not image hydrogen contained in solids such as ice crystals or the $\mathrm{CO}_{2}$ hydrates because of their much shorter transverse relaxation times. The detailed information for the MRI experiments was provided in previous work [27].

Figure 6. Schematic diagram of the MRI apparatus and the distribution of glass beads in the high pressure vessel.

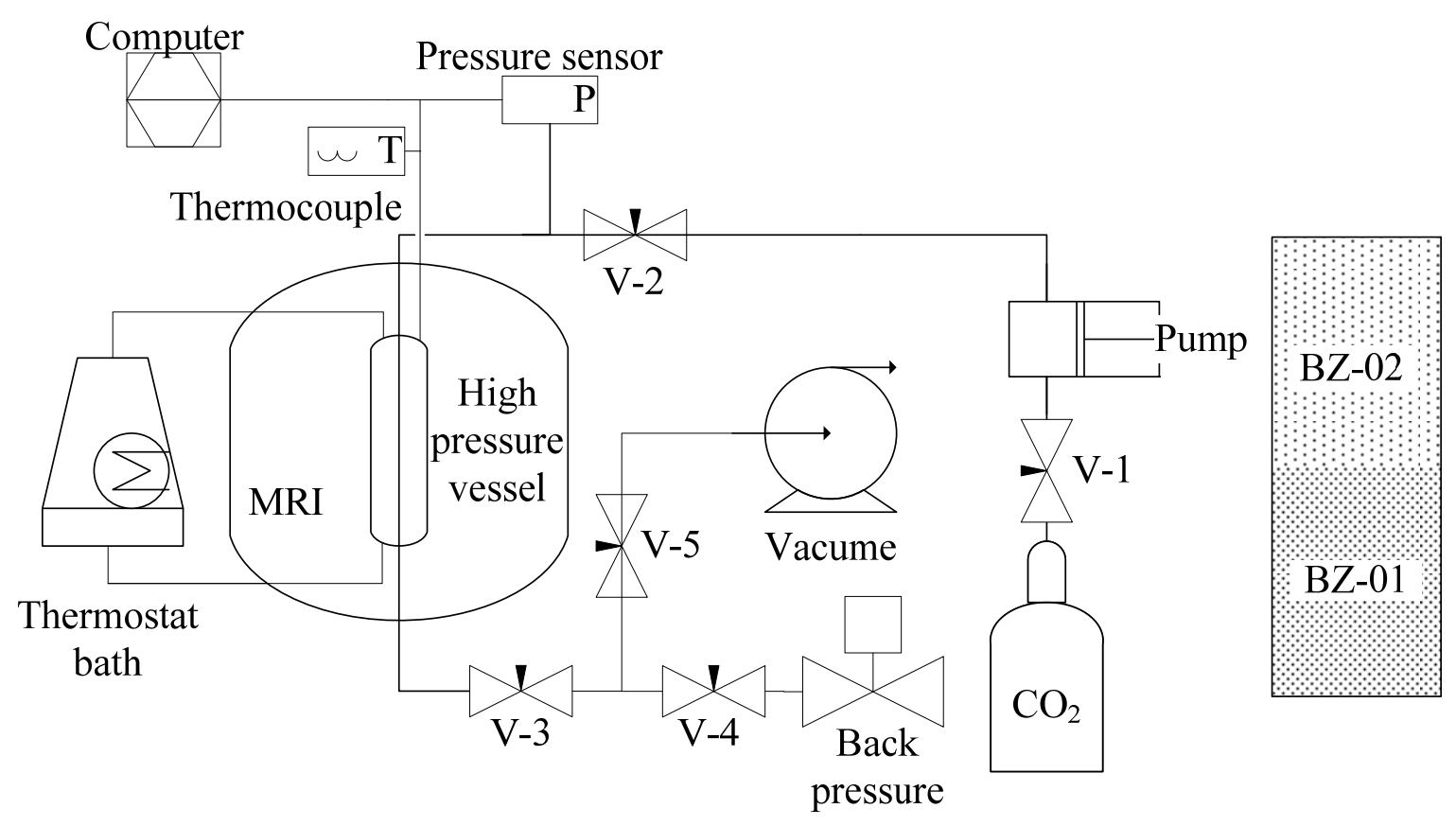


Images were obtained by using a fast spin-echo sequence, and the field of view was set to $40 \times 40 \times 40 \mathrm{~mm}$. Once the water existed in the vessel as liquid, the water distribution zone was bright in images obtained by MRI. When the water converted to hydrate (solid phase), the MRI cannot detect the water signals and the image becomes dark, so the sites that changed to dark in the image mean the formation of hydrate. Considering the randomicity of induction time, the experiment was carried out twice, the results are shown in Figure 7, and all of them indicate the $\mathrm{CO}_{2}$ hydrate formed firstly in BZ-02 (the bigger glass beads) under these experimental conditions.

Figure 7. $\mathrm{CO}_{2}$ hydrate formation process in BZ-01 and BZ-02 using MRI: (a) the first cycle; (b) the second cycle.
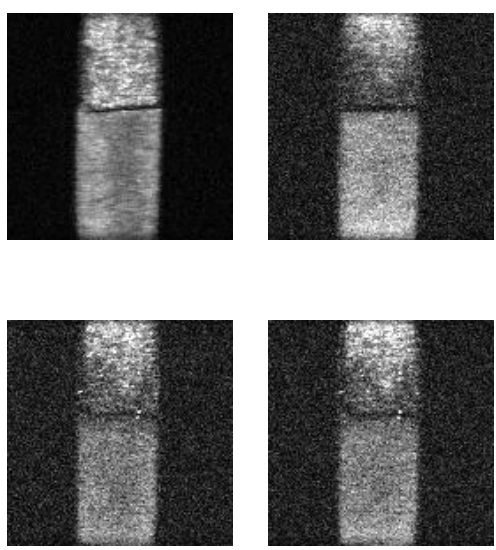
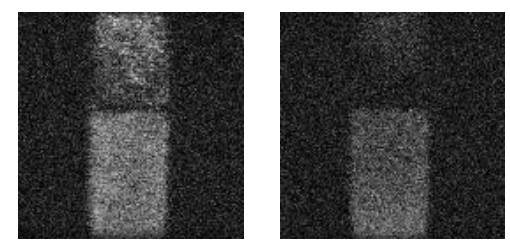

(a)
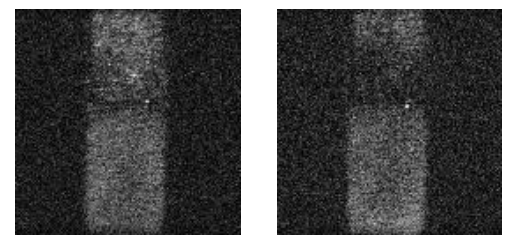

(b)
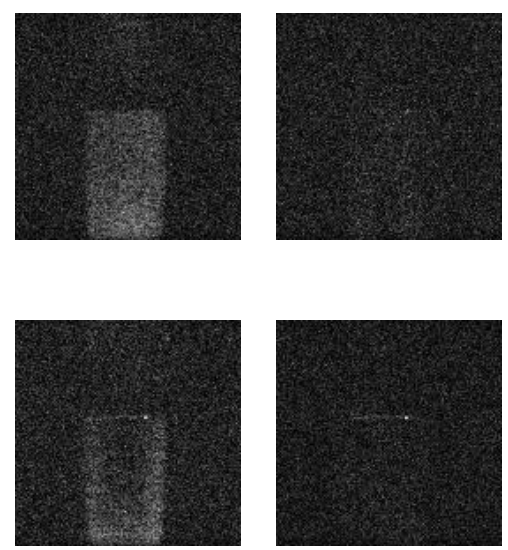

\subsection{Effects of Pores Size on $\mathrm{CO}_{2}$ Hydrate Equilibrium Condition}

The presence of porous medium affected the phase equilibrium conditions of the $\mathrm{CO}_{2}$-water-hydrate system. As shown in Figure 8, the hydrate equilibrium curve displayed a movement in the $p$ - $T$ diagram with the presence of glass beads and silica gel. The experimental data of $\mathrm{CO}_{2}$ hydrate in bulk water obtained by Deaton et al., and the experimental data of $\mathrm{CO}_{2}$ hydrate in porous medium with nominal $5.0 \mathrm{~nm}$ radii obtained by Smith et al. were quoted to compare with our data. The comparison clearly showed that the presence of porous medium with small-diameter pores affected the $\mathrm{CO}_{2}$ hydrate equilibrium conditions when compared with that in bulk water. In other words, the capillary inhibition of porous medium makes the $\mathrm{CO}_{2}$ hydrate equilibrium pressure increased at a certain temperature. The effects were mainly caused by the additional resistance effect of capillary surface tension, which leads to lower water activity and affects hydrate equilibrium condition [28]. It is important to address the discrepancy of activity between bulk water and pore-water for understanding the hydrate equilibrium pressure increase in silica gel pores and glass beads pores reported in these study. By accounting for the effects of the pore size distribution on additional forces, we can conclude that the effect of the capillary force is to lower the activity of water in the pores [29]. Once the pore size was obtained, the equilibrium pressure of $\mathrm{CO}_{2}$ hydrate in porous medium can be calculated. Based on the simulated theory, the increase of pore size caused the equilibrium pressure decrease as temperature was kept constant. When the pore size increased to some value, the effects of capillary force on hydrate equilibrium conditions becomes very small and can be ignored. The investigation of Turner et al. [23] 
indicated that the equilibrium temperature changes cannot be detected by the thermocouples in their equilibrium apparatus when the pores radius was larger than $600 \AA$.

Figure 8. Measurement of equilibrium condition for $\mathrm{CO}_{2}$ hydrate in different porous medium.

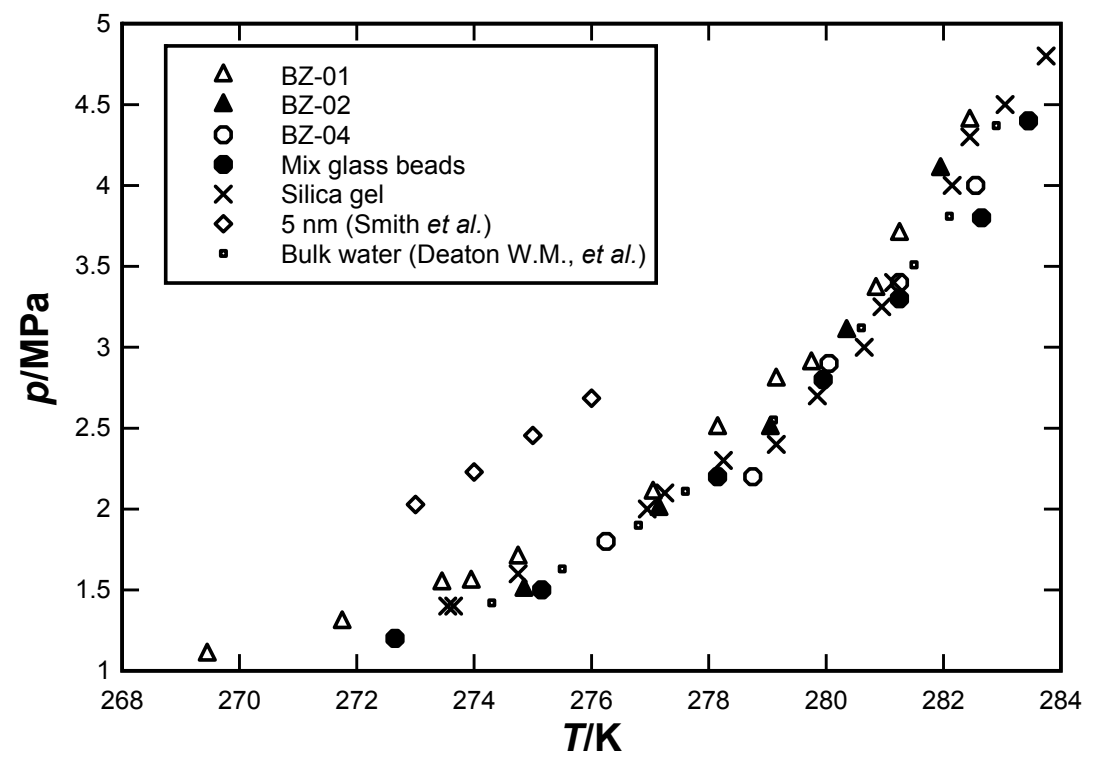

\subsection{Prediction of Hydrate Equilibrium Condition by the Improved Model}

The improved model of Song et al. [30] was used for predicting the equilibrium conditions for $\mathrm{CO}_{2}$ hydrates in porous medium, which was based on the traditional model of van der Waals and Plateeuw [31]. In this model, the mechanical equilibrium of force between the interfaces in hydrate-liquid-vapor system was considered. The solubility of $\mathrm{CO}_{2}$ gas was calculated using an empirical modification of Krichevsky-Kasarnovsky $(\mathrm{K}-\mathrm{K})$ equation [32]. This equation also includes the effect of pressure on the solubility. Having calculated the $X_{\mathrm{gas}}, X_{\mathrm{w}}$ is calculated from:

$$
X_{\mathrm{w}}=1-X_{\text {gas }}=1-f_{\text {gas }} /\left[\eta_{3} \exp \left(p^{\eta_{1}}-\eta_{2} / R T\right)\right]
$$

where $\eta_{1}, \eta_{2}, \eta_{3}$ are equal to $0.3411,22.33$ and 899683.5 for $\mathrm{CO}_{2}$, which can be found in the study of Nasrifar et al. [32].

Figure 9 shows the comparison between the experimental data and the prediction results. Considering the complexity of the system, the predictions show an acceptable agreement with the experimental data. The absolute average deviation of predicted temperature $\left(\Delta_{\mathrm{AADT}}\right)$ and pressure $\left(\Delta_{\text {AADP }}\right)$ are defined as follows [33]:

$$
\begin{aligned}
& \Delta_{\text {AADT }}=\left(1 / N_{\mathrm{p}}\right) \sum_{j=1}^{N_{\mathrm{p}}}\left[\left|T_{\text {cal }}-T_{\exp }\right| / T_{\text {exp }}\right]_{j} \times 100 \\
& \Delta_{\text {AADT }}=\left(1 / N_{\mathrm{p}}\right) \sum_{j=1}^{N_{\mathrm{p}}}\left[\left|p_{\text {cal }}-p_{\text {exp }}\right| / p_{\text {exp }}\right]_{j} \times 100
\end{aligned}
$$

where $N_{\mathrm{p}}$ denotes the number of data points. 
Figure 9. Comparison of measured and calculated equilibrium condition data for $\mathrm{CO}_{2}$ hydrate in different porous media.

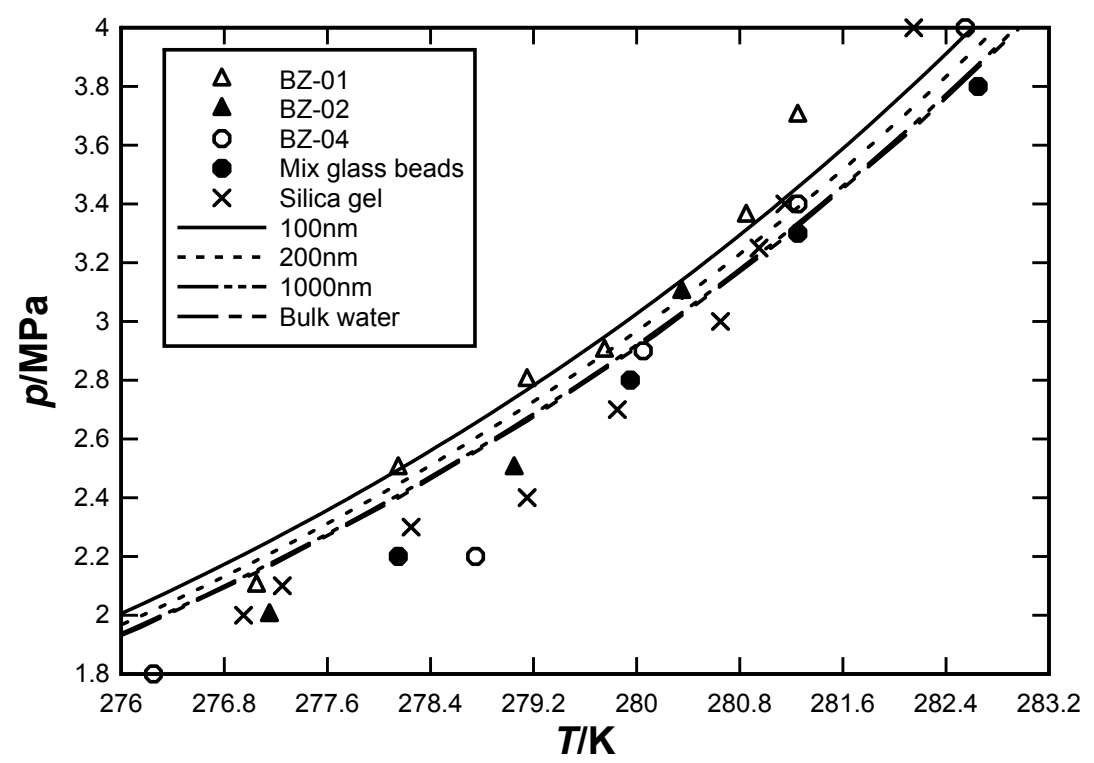

The error analysis of the predicted $\mathrm{CO}_{2}$ hydrate equilibrium conditions for different porous media is shown in Table 2.

Table 2. Absolute average deviations of predicted $\mathrm{CO}_{2}$ hydrate formation conditions.

\begin{tabular}{llllll}
\hline Porous medium & T range/K & P range/MPa & Np & AADT (\%) & AADP (\%) \\
\hline BZ-01 & $273.1-281.8$ & $1.6-3.6$ & 10 & 0.14 & 4.10 \\
BZ-02 & $274.8-282.0$ & $1.5-4.1$ & 5 & 0.32 & 9.29 \\
BZ-04 $^{*}$ & $276.2-282.8$ & $1.8-4.0$ & 5 & 0.21 & 5.36 \\
BZ-04 $^{* *}$ & $276.2-282.8$ & $1.8-4.0$ & 5 & 0.30 & 6.83 \\
Mix glass beads $^{*}$ & $275.2-282.7$ & $1.5-3.8$ & 5 & 0.20 & 8.78 \\
Mix glass beads $^{* *}$ & $275.2-282.7$ & $1.5-3.8$ & 5 & 0.24 & 9.38 \\
Silica gel $^{*}$ & $274.8-282.2$ & $1.6-4.0$ & 10 & 0.20 & 5.82 \\
Silica gel $^{* *}$ & $274.8-282.2$ & $1.6-4.0$ & 10 & 0.19 & 5.78 \\
\hline
\end{tabular}

${ }^{*}$ comparison with calculation data of bulk water; ${ }^{* *}$ comparison with calculation data of $1000 \mathrm{~nm}$.

The overall $\Delta_{\mathrm{AADT}}$ for the improved model was $0.32 \%$, respectively, and $\Delta_{\mathrm{AADP}}$ were usually less $9.38 \%$. There is a significant deviation between the measurements and the modeling results under both high pressure and low pressure conditions. However, this is not surprising due to the complexity of the system. The result that can be concluded without doubt is that the improved model gave good predictions for the $\mathrm{CO}_{2}$ hydrate in porous media.

\section{Conclusions}

Experimental and calculated phase equilibrium conditions of carbon dioxide $\left(\mathrm{CO}_{2}\right)$ hydrate in porous media were investigated in this study. When $\mathrm{CO}_{2}$ hydrate is formed in sediments with uniform pore size distribution, there is only one hydrate formation stage. When $\mathrm{CO}_{2}$ hydrate is formed in sediments with non-uniform pore size distribution, there are two hydrate formation stages, and the 
MRI apparatus results with spin-echo sequence showed that the hydrate formed firstly in BZ-02 glass beads with the same pressure and temperature. The smaller the pore size corresponds to the lower hydrate equilibrium temperature at the same pressure. This was mainly caused by the additional resistance effect of capillary surface tension. An improved model, based on the traditional model of van der Waals and Plateeuw, was used to predict $\mathrm{CO}_{2}$ hydrate equilibrium conditions, and the predictions showed good agreement with our experimental measurements.

\section{Acknowledgments}

This project is financially supported by Key program of National Natural Science Foundation of China (50736001), the National Natural Science Foundation of China (51106018), the High-tech Research and Development Program of China (2006AA09A209-5), the Major State Basic Research Development Program of China (2009CB219507), the China Postdoctoral Science Foundation (2011M500553), the Scientific Research Foundation for Doctors of Liaoning Province (20111026) and the Fundamental Research Funds for the Central Universities of China.

\section{References}

1. Ji, Y.H.; Ji, X.Y.; Feng, X.; Liu, C.; Lv, L.H.; Lu, X.H. Progress in the study on the phase equilibria of the $\mathrm{CO}_{2}-\mathrm{H}_{2} \mathrm{O}$ and $\mathrm{CO}_{2}-\mathrm{H}_{2} \mathrm{O}-\mathrm{NaCl}$ systems. Chin. J. Chem. Eng. 2007, 15, 439-448.

2. Bachu, S. Sequestration of $\mathrm{CO}_{2}$ in geological media: Criteria and approach for site selection in response to climate change. Energy Convers. Manag. 2000, 41, 953-970.

3. Unander, F. Energy Technology Perspectives: Scenarios and Strategies to 2050; Technical Report; International Energy Agency: Paris, France, 2008.

4. Sloan, E.D. Clathrate Hydrates of Natural Gases, 2nd ed.; Marcel Dekker Inc.: New York, NY, USA, 1998.

5. Yang, H.Q.; Xu, Z.H.; Fan, M.H.; Gupta, R.; Slimane, R.B.; Bland, A.E.; Wright, I. Progress in carbon dioxide separation and capture: A review. J. Environ. Sci. 2008, 20, 14-27.

6. Brewer, P.G.; Orr, F.M., Jr.; Friederich, G.; Kvenvolden, K.A.; Orange, D.L. Gas hydrate formation in the deep sea: In situ experiments with controlled release of methane, natural gas and carbon dioxide. Energy Fuels 1998, 12, 183-188.

7. Wendland, M.; Hasse, H.; Maurer, G. Experimental pressure-temperature data on three- and four-phase equilibria of fluid, hydrate and ice phases in the system carbon dioxide water. J. Chem. Eng. Data 1999, 44, 901-906.

8. Yang, S.O.; Yang, I.M.; Kim, Y.S.; Lee, C.S. Measurement and prediction of phase equilibria for water $+\mathrm{CO}_{2}$ mixture in hydrate forming conditions. Fluid Phase Equilib. 2000, 175, 75-89.

9. Englezos, P.; Hall, S. Phase equilibrium data on carbon dioxide hydrate in the presence of electrolytes, water soluble polymers and montmorillonite. Can. J. Chem. Eng. 1994, 72, 887-893.

10. Breland, E.; Englezos, P. Equilibrium hydrate formation data for carbon dioxide in aqueous glycerol solutions. J. Chem. Eng. Data 1996, 41, 11-13.

11. Dholabhai, P.D.; Scott Parent, J.; Raj Bishnoi, P. Carbon dioxide hydrate equilibrium conditions in aqueous solutions containing electrolytes and methanol using a new apparatus. Ind. Eng. Chem. Res. 1996, 35, 819-823. 
12. Dholabhai, P.D.; Scott Parent, J.; Raj Bishnoi, P. Equilibrium conditions for hydrate formation from binary mixtures of methane and carbon dioxide in the presence of electrolytes, methanol and ethylene glycol. Fluid Phase Equilib. 1997, 41, 235-246.

13. Kang, S.P.; Chun, M.K.; Lee, H. Phase equilibria of methane and carbon dioxide hydrates in the aqueous $\mathrm{MgCl}_{2}$ solutions. Fluid Phase Equilib. 1998, 147, 229-238.

14. Mohammadi, A.H.; Afzal, W.; Richon, D. Gas hydrates of methane, ethane, propane and carbon dioxide in the presence of single $\mathrm{NaCl}, \mathrm{KCl}$ and $\mathrm{CaCl}_{2}$ aqueous solutions: Experimental measurements and predictions of dissociation conditions. J. Chem. Thermodyn. 2008, 40, 1693-1697.

15. Handa, Y.P.; Stupin, D.Y. Thermodynamic properties and dissociation characteristics of methane and propane hydrates in 70-Å-radius silica gel pores. J. Phys. Chem. 1992, 96, 8599-8603.

16. Uchida, T.; Ebinuma, T.; Ishizaki, T. Dissociation condition measurements of methane hydrate in confined small pores of porous glass. J. Phys. Chem. B 1999, 103, 3659-3662.

17. Clennell, M.B.; Hovland, M.; Booth, J.S.; Henry, P.; Winters, W.J. Formation of natural gas hydrates in marine sediments 1: Conceptual model of gas hydrate growth conditioned by host sediment properties. J. Geophys. Res. 1999, 104, 22985-23003.

18. Henry, P.; Thomas, M.; Clennell, M.B. Formation of natural gas hydrates in marine sediments 2 : Thermodynamic calculations of stability conditions in porous sediments. J. Geophys. Res. 1999, 104, 23005-23022.

19. Zatsepina, O.Y.; Buffet, B.A. Nucleation of $\mathrm{CO}_{2}$ hydrate in porous medium. Fluid Phase Equilib. 2002, 200, 263-275.

20. Smith, D.; Wilder, J.; Seshadri, K. Thermodynamics of carbon dioxide hydrate formation in media with broad pore-size distributions. Environ. Sci. Technol. 2002, 36, 5192-5198.

21. Anderson, R.; Llamedo, M.; Tohidi, B.; Burgass, R.W. Experimental measurement of methane and carbon dioxide clathrate hydrate equilibria in mesoporous silica. J. Phys. Chem. B. 2003, 107, 3507-3514.

22. Kumar, A. Formation and Dissociation of Gas Hydrates in Porous Media. Master Thesis, University of Calgary, Calgary, Canada, 2005.

23. Turner, D.J.; Cherry, R.S.; Sloan, E.D. Sensitivity of methane hydrate phase equilibria to sediment pore size. Fluid Phase Equilib. 2005, 228-229, 505-510.

24. Aladko, E.Y.; Dyadin, Y.A.; Fenelonov, V.B.; Larionov, E.G.; Manakov, A.Y.; Mel'gunov, M.S.; Zhurko, F.V. Formation and decomposition of ethane, propane, and carbon dioxide hydrates in silica gel mesopores under high pressure. J. Phys. Chem. B 2006, 110, 19717-19725.

25. Song, Y.C.; Yang, M.J.; Liu, Y.; Li, Q.P. Influence of ions on phase equilibrium of methane hydrate. CIESC J. 2009, 60, 1362-1366.

26. Yang, M.J.; Song, Y.C.; Liu, Y.; Chen, Y.J.; Li, Q.P. Influence of pore size, salinity and gas composition upon the hydrate formation conditions. Chin. J. Chem. Eng. 2010, 18, 292-296.

27. Yang, M.J.; Song, Y.C.; Zhao, Y.C.; Liu, Y.; Jiang, L.L.; Li, Q.P. MRI measurements of $\mathrm{CO}_{2}$ hydrate dissociation rate in a porous medium. Magn. Reson. Imaging 2011, 29, 1007-1013.

28. Wilder, J.W.; Seshadri, K.; Smith, D.H. Modeling hydrate formation in media with broad pore size distributions. Langmuir 2001, 17, 6729-6735. 
29. Clarke, M.A.; Darvish, M.P.; Bishnoi, P.R. A method to predict equilibrium conditions of gas hydrate formation in porous media. Ind. Eng. Chem. Res. 1999, 38, 2485-2490.

30. Song, Y.C.; Yang, M.J.; Chen, Y.J.; Li, Q.P. An improved model for predicting hydrate phase equilibrium in marine sediment environment. J. Nat. Gas Chem. 2010, 19, 241-245.

31. Van der Waals, J.H.; Platteeuw, J.C. Clathrate solution. Adv. Chem. Phys. 1959, 2, 1-57.

32. Nasrifar, K. A model for prediction of gas hydrate formation conditions in aqueous solutions containing electrolytes and/or alcohol. J. Chem. Thermodyn. 2001, 33, 999-1014.

33. Mei, D.H.; Liao, J.; Yang, J.T.; Guo, T.M. Prediction of equilibrium hydrate formation conditions in electrolyte aqueous solutions. Acta Petrolei Sinica 1998, 14, 86-93.

(C) 2012 by the authors; licensee MDPI, Basel, Switzerland. This article is an open access article distributed under the terms and conditions of the Creative Commons Attribution license (http://creativecommons.org/licenses/by/3.0/). 